\title{
Ways to increase the probability of acceptance of a manuscript
}

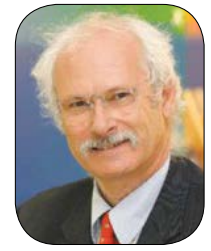

Jean-François ROULET

DDS, Habil, Prof hc, Drhc, Professor

Editor-in-Chief

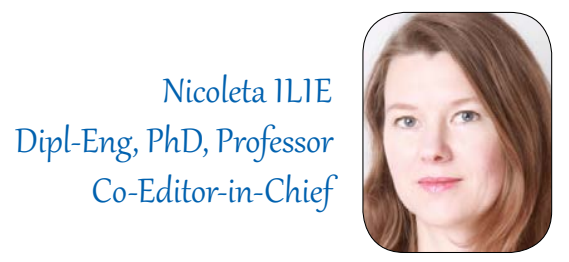

Dear Authors,

You may think writing a scientific manuscript is difficult. Providing you had a well-designed experiment based on a wellthought through question, it is quite simple, since it is a standard procedure. How to get it through the review process is a vital question for you, the author. The answer is very simple: please the editor and the reviewers. There is nothing more boring for editors and reviewers to make the same corrections again and again.

Therefore, here is a list of the most common errors we see in our editorial work:

The most prominent reasons for rejection or a straight route to revision are manuscripts that have a poor language or do not comply with the instructions for authors. We ask ourselves whether it is so difficult to read instructions and follow them. Not doing so at least prolongs the review process considerably. Most of us publish not in our mother tongue but in English. Ergo have an expert in dental research and English mother tongue look over your manuscript. If you do not know any, then look for some professional scientific editing service. The internet may help.

Thinking about Materials and Methods, we are convinced that they should be described as detailed as possible so that someone could replicate the experiment. If in factorial designs the number of samples divided by the $n$ (samples per group) does not end up in a full number, we do not know what you did and usually stop reading. The reviewers and the readers of a paper must perfectly understand the experimental design and what was done in order to judge what was done and if it was done correctly. We highly recommend to use flow charts and figures to explain the experiments performed. To disclose your sources and compositions of materials is mandatory.

Statistics are very often the reason for rejection. Please describe exactly the statistical tools you have used to analyze the data. Very often not appropriate tests are used. Furthermore, add to all tables and figures self-explanatory legends and the information about statistics. In two-way designs tell the readers about significant interactions and if needed differentiate between rows and columns. Make sure you distinguish between normally distributed data and data with skewed distributions. Do NOT mix both statistical worlds (e.g. analyze with ANOVA, but report data as box plots). Finally, strictly avoid to talk about differences between groups that are not statistically significantly different. Formulations like "group D showed the highest values, but there was no significant difference between Groups B, C, D, and E" are an absolute no go. It is also very questionable to talk about "trends" if no significant differences were found. Consulting a statistician is a very good idea that may pay off big.

Discussions are sometimes very simplistic or difficult to follow. Structuring the discussion into a discussion of the material and methods, followed by the discussion of the results helps to follow your thoughts. Try to explain why you did that, 
explain what the results mean, and why they are different from the results of your peers. Please associate literature quotes to your explanations and make it clear which are facts and which are hypothetical explanations.

Finally, base your conclusions only on the results of your experiment and do not include your wishful thinking.

The references are proof that you know the actual literature and are an expert in the field. It is without question that they must be updated and complete. Furthermore, every quoted paper must be listed and every listed paper must be quoted.

It seems to us that what is said above is quite straight forward. Be assured that good editing is meticulously adhering to such facts in order to allow the readers of the Stomatology Edu Journal (Stoma Edu J) to understand what was done and to make their judgement, if it is solid and how it may influence their research or clinical practice.

Sincerely yours,

$\begin{array}{ll}\text { J.-F. Roulet } & \text { N. Ilie } \\ \text { Editor-in-Chief } & \text { Co-Editor-in-Chief }\end{array}$

DOI: https://doi.org/10.25241/stomaeduj.2019.6(3).edit.2 